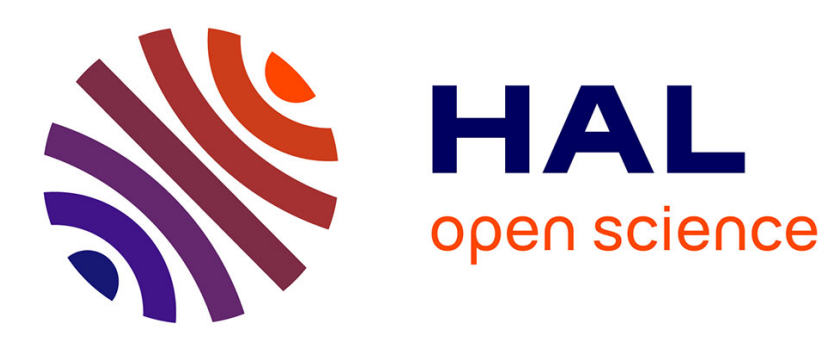

\title{
Homogeneous finite time observer for nonlinear systems with linearizable error dynamics
}

\author{
Wilfrid Perruquetti, Thierry Floquet
}

\section{To cite this version:}

Wilfrid Perruquetti, Thierry Floquet. Homogeneous finite time observer for nonlinear systems with linearizable error dynamics. IEEE Conference on Decision and Control, Dec 2007, New Orleans, United States. 10.1109/CDC.2007.4434702 . inria-00171342

\section{HAL Id: inria-00171342 \\ https://hal.inria.fr/inria-00171342}

Submitted on 12 Sep 2007

HAL is a multi-disciplinary open access archive for the deposit and dissemination of scientific research documents, whether they are published or not. The documents may come from teaching and research institutions in France or abroad, or from public or private research centers.
L'archive ouverte pluridisciplinaire HAL, est destinée au dépôt et à la diffusion de documents scientifiques de niveau recherche, publiés ou non, émanant des établissements d'enseignement et de recherche français ou étrangers, des laboratoires publics ou privés. 


\title{
Homogeneous finite time observer for nonlinear systems with linearizable error dynamics
}

\author{
W. Perruquetti and T. Floquet
}

\begin{abstract}
This paper introduces a finite time observer for nonlinear systems that can be put into a linear canonical form up to output injection. The main contribution is that finite time observation is obtained using continuous output injections. The method is applied to a problem of chaotic synchronization.
\end{abstract}

\section{INTRODUCTION}

Several approaches have been considered to design observers for nonlinear systems. One of them is to study the possibility to transform the original nonlinear system into some observer canonical forms that admit observer error linearization. The linearization by input-output injection, that consists in finding an equivalent observable linear system up to output injection, has been studied in [6], [14], [25], [26], [29], [42]. Extensions were given in [15], [38] using output dependent time scaling, and in [2], [23] using system immersion. Then, Luenberger based linear observer with asymptotically stable error dynamics can be designed.

The purpose of this paper is to introduce an homogeneous observer for nonlinear systems that are linearizable up to output injection. This observer yields the finite time convergence of the error variables. Whereas finite time convergence can be usually obtained using discontinuous actions and their successive filtered values, the observer given in this brief only relies on continuous homogeneous output injections. Thus, high frequency dynamics are avoided and low pass filters, that could introduce delays in the estimate, are not required. It is also shown that the Luenberger linear observer and the higher order sliding mode differentiator introduced in [27] are limit cases of the proposed observer in this paper.

The paper is organized as follows. The class of considered systems is given in Section II. Notions of finite time stability and the design of a continuous finite time observer are presented in Section III. In Section IV, the link to finite time differentiators is discussed. Finally, Section V gives an example.

\section{Problem Statement}

Let us consider the following ordinary differential equation:

$$
\dot{x}=g(x), \quad x \in \mathbb{R}^{n} .
$$

This work is supported in part by the Region Nord Pas-de-Calais and the FEDER Fonds Européen de Déeveloppement Régional (European Funds of Regional Development) under the project AUTORIS-TAT T31. W. Perruquetti and T. Floquet are with LAGIS UMR CNRS 8146, Ecole Centrale de Lille, BP 48, Cité Scientifique, 59651 Villeneuve-d'Ascq, France, and with Projet ALIEN, INRIA Futurs. wilfrid.perruquetti; thierry.floquetdec-lille.fr
Note $\phi^{x_{0}}(t)$ a solution of the system (1) starting from $x_{0}$ at time zero.

If $g$ is a continuous but not Lipschitz function, it may happen that any solution of (1) converges to zero in finite time. For instance, it is the case for

$$
\dot{x}=-\operatorname{sign}(x)|x|^{\frac{1}{3}}, \quad x \in \mathbb{R}
$$

whose solutions are

$$
\begin{aligned}
& \phi^{x_{0}}(t)=\operatorname{sign}\left(x_{0}\right)\left(\left|x_{0}\right|^{\frac{1}{3}}-\frac{t}{3}\right)^{3}, \text { if } 0<t<3\left|x_{0}\right|^{\frac{1}{3}} \\
& \phi^{x_{0}}(t)=0, \text { if } t \geq 3\left|x_{0}\right|^{\frac{1}{3}},
\end{aligned}
$$

and tends to zero in finite time. It is aimed here to exploit this property of dynamical systems to design a finite time observer (FTO).

Let us consider a nonlinear system of the form:

$$
\begin{aligned}
& \dot{\xi}=\eta(\xi, u) \\
& y=h(\xi)
\end{aligned}
$$

where $\xi \in \mathbb{R}^{d}$ is the state, $u \in \mathbb{R}^{m}$ is a known and sufficiently smooth control input, and $y(t) \in \mathbb{R}$ is the output. $\eta: \mathbb{R}^{d} \times \mathbb{R}^{m} \rightarrow \mathbb{R}^{d}$ is a known vector field. It is assumed that the system (2)-(3) is locally observable [17] and that there exists a local state coordinate transformation and an output coordinate transformation which transform the nonlinear system (2)-(3) into the following canonical form:

$$
\begin{aligned}
\dot{x} & =A x+f\left(y, u, \dot{u}, \ldots, u^{(r)}\right) \\
y & =C x
\end{aligned}
$$

where $x \in \mathbb{R}^{n}$ is the state, $r \in \mathbb{N}_{>0}$ and

$$
\begin{aligned}
A & =\left(\begin{array}{ccccc}
0 & 1 & 0 & 0 & 0 \\
0 & 0 & 1 & 0 & 0 \\
\vdots & \vdots & \vdots & \ddots & \vdots \\
0 & 0 & 0 & 0 & 1 \\
0 & 0 & 0 & 0 & 0
\end{array}\right), \\
C & =\left(\begin{array}{cccc}
1 & 0 & \ldots & 0
\end{array}\right) .
\end{aligned}
$$

The transformations involved in such a linearization method for different classes of systems can be found in [6], [14], [25], [26], [29], [42]. Usually, $n=d$ but one can have $n>d$ in the case of system immersion [2], [23].

Then, the observer design is quite simple since all nonlinearities are function of the output and known inputs. Asymptotic stability can be obtained using a straightforward generalization of a linear Luenberger observer. Finite time sliding mode observers have already been designed for 
system (4)-(5) (see e.g. [12], [35]). However, they rely on discontinuous output injections and on a step-by-step procedure that can be harmful for high order systems. In this paper, a finite time observer based on continuous output injections is introduced.

\section{HomogeneOUS FINITE TIME OBSERVER}

\section{A. Definitions and preliminary results}

1) Finite time stability:

Definition 1: The system (1) is said to have unique solutions in forward time on a neighbourhood $\mathcal{U} \subset \mathbb{R}^{n}$ if for any $x_{0} \in \mathcal{U}$ and two right maximally defined solutions of (1), $\phi^{x_{0}}:\left[0, T_{\phi}\left[\rightarrow \mathbb{R}^{n}\right.\right.$ and $\psi^{x_{0}}:\left[0, T_{\psi}\left[\rightarrow \mathbb{R}^{n}\right.\right.$, there exists $0<T_{x_{0}} \leq \min \left\{T_{\phi}, T_{\psi}\right\}$ such that $\phi^{x_{0}}(t)=\psi^{x_{0}}(t)$ for all $t \in\left[0, T_{x_{0}}[\right.$.

It can be assumed that for each $x_{0} \in \mathcal{U}, T_{x_{0}}$ is chosen to be the largest in $\mathbb{R}_{+} \cup\{+\infty\}$. Various sufficient conditions for forward uniqueness can be found in [22].

Let us consider the system (1) where $g \in C^{0}\left(\mathbb{R}^{n}\right), g(0)=$ 0 and where $g$ has unique solutions in forward time. Let us recall the notion of finite time stability involving the settlingtime function given in [5, Definition 2.2] and [1].

Definition 2: The origin of the system (1) is finite time stable if:

1) there exists a function $T: \mathcal{V} \backslash\{0\} \rightarrow \mathbb{R}_{+}(\mathcal{V}$ is a neighbourhood of the origin) such that for all $x_{0} \in \mathcal{V} \backslash\{0\}, \phi^{x_{0}}(t)$ is defined (and unique) on $\left[0, T\left(x_{0}\right)\right), \phi^{x_{0}}(t) \in \mathcal{V} \backslash\{0\}$ for all $t \in\left[0, T\left(x_{0}\right)\right)$ and $\lim _{t \rightarrow T\left(x_{0}\right)} \phi^{x_{0}}(t)=0$.

$T$ is called the settling-time function of the system (1).

2) for all $\epsilon>0$, there exists $\delta(\epsilon)>0$ such that for every $x_{0} \in\left(\delta(\epsilon) \mathcal{B}^{n} \backslash\{0\}\right) \cap \mathcal{V}, \phi^{x_{0}}(t) \in \epsilon \mathcal{B}^{n}$ for all $t \in\left[0, T\left(x_{0}\right)\right)$.

Remark 3: Note that if the origin of the system (1) is finite time stable, then $g$ cannot have unique solutions in backward time at the origin. In particular, $g$ cannot be locally Lipschitz at the origin (see the example given in the problem statement Section II). Then, if the system (1) is finite time stable, Lyapunov asymptotic stability implies that $\phi^{0} \equiv 0$ is the unique solution starting from $x_{0}=0$. So, the settling-time $T$ can be extended at the origin by $T(0)=0$. This extension is also called the settling-time function of the system (1).

The following result gives a sufficient condition for system (1) to be $\mathrm{FTS}^{1}$ (see [31], [36] for ODE, and [30] for Differential inclusion):

Theorem 4: Let the origin be an equilibrium point for the system (1), and let $\varphi$ be continuous on an open neighborhood $\mathcal{V}$ of the origin. If there exist a Lyapunov function ${ }^{2} V: \mathcal{V} \rightarrow$ $\mathbb{R}_{+}$and a function $r: \mathbb{R}_{+} \rightarrow \mathbb{R}_{+}$such that

$$
\dot{V}(x) \leq-r(V(x)),
$$

\footnotetext{
${ }^{1}$ This result is based on a necessary and sufficient condition given in [16] for scalar system in the form (1).

${ }^{2} V$ is a continuously differentiable function defined on $\mathcal{V}$ such that $V$ is positive definite and $V$ is negative definite.
}

along the solutions of (1) and $\varepsilon>0$ such that

$$
\int_{0}^{\varepsilon} \frac{d z}{r(z)}<+\infty
$$

then the origin is FTS.

In particular, assuming forward uniqueness of the solution and the continuity of the settling time function, Bhat and Bernstein (see [5, Definition 2.2]) showed that "finite time stability of the origin is equivalent to the existence of a Lyapunov function satisfying (7) where $r(x)=c x^{a}$, with $a \in] 0,1[, c>0$ ".

The interested reader can find more details on finite time stability in [1], [3], [4], [5], [16], [19], [20], [31], [30], [33].

\section{2) Homogeneity:}

Definition 5: Let $r=\left(r_{1}, \ldots, r_{n}\right)$ be a $n$-uplet of positive real numbers. Then for any positive real number $\lambda$

$$
\Lambda_{r} x=\left(\ldots, \lambda^{r_{i}} x_{i}, \ldots\right)
$$

represents a mapping $x \mapsto \Lambda_{r} x$ usually called dilation (see [18]).

Definition 6: A function $h$ defined on $\mathbb{R}^{n}$ is said to be homogeneous with degree $\alpha_{h} \in \mathbb{R}$ with respect to dilation $\Lambda_{r}$ if for all $x \in \mathbb{R}^{n}$ (see [18]):

$$
h\left(\Lambda_{r} x\right)=\lambda^{\alpha_{h}} h(x) .
$$

When such a property holds, we note: $\operatorname{deg}(h)=\alpha_{h}$.

Definition 7: A vector field $g$ defined on $\mathbb{R}^{n}$ with components denoted by $g_{i}$ is said to be homogeneous with degree $d$ with respect to dilation $\Lambda_{r}$ (with $r=\left(r_{1}, \ldots, r_{n}\right)$ ) if for all $x \in \mathbb{R}^{n}$, (see [18]):

$$
\operatorname{deg}\left(g_{i}\right)=d+r_{i}, \forall i \in\{1, \ldots, n\}
$$

When such a property holds, the corresponding nonlinear ODE given by (1) is said to be homogeneous with degree $d$ with respect to dilation $\Lambda_{r}$.

Theorem 8: [1, Theorem 5.8 and Corollary 5.4] Let $g$ be defined on $\mathbb{R}^{n}$ and be a continuous vector field homogeneous with degree $d<0$ (with respect to dilation $\Lambda_{r}$ ). If the origin of (1) is locally asymptotically stable, it is globally FTS.

\section{B. Finite Time Observer design}

Set $x=\left[\begin{array}{llll}x_{1} & x_{2} & \cdots & x_{n}\end{array}\right]^{T}$. The system (4)-(5) can be rewritten as:

$$
\begin{aligned}
\dot{x}_{1} & =x_{2}+f_{1}\left(x_{1}, u, \dot{u}, \ldots, u^{(r)}\right) \\
\dot{x}_{2} & =x_{3}+f_{2}\left(x_{1}, u, \dot{u}, \ldots, u^{(r)}\right) \\
& \vdots \\
\dot{x}_{n} & =f_{n}\left(x_{1}, u, \dot{u}, \ldots, u^{(r)}\right) \\
y & =x_{1}
\end{aligned}
$$


The observer is designed as follows:

$$
\begin{aligned}
\frac{d \widehat{x}_{1}}{d t} & =\widehat{x}_{2}+f_{1}\left(x_{1}, u, \dot{u}, \ldots, u^{(r)}\right)+\chi_{1}\left(x_{1}-\widehat{x}_{1}\right) \\
\frac{d \widehat{x}_{2}}{d t} & =\widehat{x}_{3}+f_{2}\left(x_{1}, u, \dot{u}, \ldots, u^{(r)}\right)+\chi_{2}\left(x_{1}-\widehat{x}_{1}\right) \\
& \vdots \\
\frac{d \widehat{x}_{n}}{d t} & =f_{n}\left(x_{1}, u, \dot{u}, \ldots, u^{(r)}\right)+\chi_{n}\left(x_{1}-\widehat{x}_{1}\right)
\end{aligned}
$$

where the functions $\chi_{i}$ will be defined in such a way that the observation error $e=x-\widehat{x} \in \mathbb{R}^{n}$ tends to zero in finite time. The error dynamics is given by

$$
\begin{gathered}
\dot{e}_{1}=e_{2}+\chi_{1}\left(e_{1}\right) \\
\dot{e}_{2}=e_{3}+\chi_{2}\left(e_{1}\right) \\
\vdots \\
\dot{e}_{n}=\chi_{n}\left(e_{1}\right)
\end{gathered}
$$

Consider a dilation with weights $\left(r_{1}, r_{2}, \ldots, r_{n}\right)$. The system (12) is homogeneous with degree $d$ if and only if the functions $\chi_{i}$ are homogeneous. Furthermore:

$$
\begin{gathered}
r_{1}+d=r_{2}=\operatorname{deg}\left(\chi_{1}\right), \\
r_{2}+d=r_{3}=\operatorname{deg}\left(\chi_{2}\right), \\
\vdots \\
r_{n}+d=\operatorname{deg}\left(\chi_{n}\right) .
\end{gathered}
$$

Let us choose $d<0$ and $\chi_{i}\left(e_{1}\right)=-k_{i}\left\lfloor e_{1}\right\rceil^{\alpha_{i}}$, where for any real number $x \in \mathbb{R}$ :

$$
\lfloor x\rceil^{\alpha}=\operatorname{sgn}(x)|x|^{\alpha} .
$$

Note that for any $\alpha>0$ :

$$
\frac{d\lfloor x\rceil^{\alpha}}{d x}=\alpha|x|^{\alpha-1}, \quad \frac{d|x|^{\alpha}}{d x}=\alpha\lfloor x\rceil^{\alpha-1} .
$$

Then $\operatorname{deg}\left(\chi_{i}\right)=\alpha_{i} r_{1}$ and

$$
\begin{aligned}
r_{1} & =\frac{r_{2}}{\alpha_{1}}=\frac{r_{3}}{\alpha_{2}}=\ldots=\frac{r_{n}}{\alpha_{n-1}}>0, \\
d & =\left(\alpha_{1}-1\right) r_{1}=\left(\frac{\alpha_{2}}{\alpha_{1}}-1\right) r_{2}=\ldots=\left(\frac{\alpha_{n}}{\alpha_{n-1}}-1\right) r_{n}<0 .
\end{aligned}
$$

This is equivalent to the following conditions on the $\alpha_{i}$ :

$$
\begin{aligned}
\alpha_{1} & =\alpha \in] \frac{n-1}{n}, 1[, \\
\alpha_{2} & =2 \alpha-1 \\
\alpha_{3} & =3 \alpha-2, \\
& \vdots \\
\alpha_{n} & =n \alpha-(n-1) .
\end{aligned}
$$

Then (12) becomes

$$
\begin{aligned}
\frac{d e_{1}}{d t} & =e_{2}-k_{1}\left\lfloor e_{1}\right\rceil^{\alpha} \\
\frac{d e_{2}}{d t} & =e_{3}-k_{2}\left\lfloor e_{1}\right\rceil^{2 \alpha-1} \\
\vdots & \\
\frac{d e_{n}}{d t} & =-k_{n}\left\lfloor e_{1}\right\rceil^{n \alpha-(n-1)} .
\end{aligned}
$$

Since the dilation is $\left(r_{1}, r_{2}=\alpha_{1} r_{1}, \ldots, r_{n}=\alpha_{n-1} r_{1}\right)$, the dilation weights can be normalized by taking $r_{1}=1$ and one obtains $\left(1, \alpha_{1}, \ldots, \alpha_{n-1}\right)$. Let us consider the following Lyapunov function:

$$
\begin{aligned}
V_{\alpha}(e) & =\sigma^{T} P \sigma \\
\sigma & =\left[\left\lfloor e_{1}\right\rceil^{1 / r_{1}},\left\lfloor e_{2}\right\rceil^{1 / r_{2}} \ldots,\left\lfloor e_{n}\right\rceil^{1 / r_{n}}\right]^{T} \\
& =\left[e_{1},\left\lfloor e_{2}\right\rceil^{1 / \alpha} \ldots,\left\lfloor e_{n}\right\rceil^{1 /((n-1) \alpha-(n-2))}\right]^{T},
\end{aligned}
$$

where $P$ is the solution of the following Lyapunov equation

$$
\begin{aligned}
A_{o}^{T} P+P A_{o} & =-I, \\
A_{o}= & \left(\begin{array}{cccc}
-k_{1} & 1 & 0 & 0 \\
-k_{2} & 0 & \ddots & 0 \\
\vdots & \vdots & \ddots & 1 \\
-k_{n} & 0 & \ldots & 0
\end{array}\right) .
\end{aligned}
$$

The gains $k_{i}$ are chosen such that $A_{o}$ is Hurwitz. Thus $P$ is positive definite. Note that $V$ is homogeneous because

$$
\begin{gathered}
\sigma\left(\Lambda_{r} e\right)=\left[\begin{array}{c}
\lambda e_{1} \\
\left\lfloor\lambda^{\alpha} e_{2}\right\rceil^{1 / \alpha} \\
\vdots \\
\left\lfloor\left.\lambda^{((n-1) \alpha-(n-2))} e_{n}\right|^{1 /((n-1) \alpha-(n-2))}\right.
\end{array}\right] \\
=\lambda \sigma(e) \\
V_{\alpha}\left(\Lambda_{r} e\right)=\sigma^{T}\left(\Lambda_{r} e\right) \operatorname{P\sigma }\left(\Lambda_{r} e\right)=\lambda^{2} V_{\alpha}(e)
\end{gathered}
$$

and that $V$ is differentiable for $0<\alpha \leq 1$ because each component of $\sigma$ is of the type $\left[e_{i}\right]^{1 / r_{i}}$ with $1 / r_{i} \geq 1$. It is clear that the previous obtained conditions are parameterized by the single parameter $\alpha$. Let us also note that when $\alpha$ tends to 1 , the following facts hold:

- $\lim _{\alpha \rightarrow 1}\left(\alpha_{i}\right)=1, \quad \forall i \in\{1, \ldots, n\}$,

- $\lim _{\alpha \rightarrow 1}\left(r_{i}\right)=r_{1}=1, \quad \forall i \in\{1, \ldots, n\}$, (after normaliz$\underset{\mathrm{ing}}{\alpha \rightarrow 1}$ the dilation $r_{1}=1$ ),

- $\lim _{\alpha \rightarrow 1}(\sigma)=\lim _{\alpha \rightarrow 1}\left[e_{1},\left\lfloor e_{2}\right\rceil^{1 / \alpha} \ldots,\left\lfloor e_{n}\right\rceil^{1 /((n-1) \alpha-(n-2))}\right]^{T}=$

- the system (14) tends to the globally asymptotically stable linear system $\dot{x}=A_{o} x$ since $A_{o}$ is Hurwitz.

Let us define the following level set

$$
L_{\alpha}=\left\{e: V_{\alpha}(e)=1\right\} .
$$

When $\alpha=1, V_{\alpha=1}$ is a positive definite quadratic Lyapunov function and its time derivative is $\dot{V}_{\alpha=1}(e)=-e^{T} e<0$. 
From the continuity of the two functions $V_{\alpha}(e)$ and $\dot{V}_{\alpha}(e)$, it can be said that, for $\alpha$ close to $1, L_{\alpha}$ is a compact set where $V_{\alpha}(e)$ is strictly positive and its time derivative is strictly negative.

From the facts that $V_{\alpha}(e)$ and $-\dot{V}_{\alpha}(e)$ are strictly positive definite on the level set $L_{\alpha}$ (that contains the origin) and the homogeneity property of both the system and the function $V_{\alpha}$, one can conclude at the asymptotic stability of the system (see [3], [24], [1]). Moreover, if $\alpha$ is chosen such that $d<0$, one can state that there exists a positive constant $\varepsilon, \frac{1}{n}>\varepsilon>$ 0 , such that the observer (11) with $\chi_{i}\left(e_{1}\right)=-k_{i}\left\lfloor e_{1}\right\rceil^{\alpha_{i}}$, $i \in\{1, \ldots, n\}$ and the following positive constants

$$
\begin{aligned}
\alpha_{1} & =\alpha \in] 1-\varepsilon, 1[, \\
\alpha_{2} & =2 \alpha-1, \\
\alpha_{3} & =3 \alpha-2, \\
& \vdots \\
\alpha_{n} & =n \alpha-(n-1), \\
k_{i} & : A_{o} \text { given by }(15) \text { is Hurwitz }
\end{aligned}
$$

reconstruct in finite time the state $x$.

\section{CONTINUOUS FINITE TIME DIFFERENTIATOR}

\section{A. Description and analysis}

From Section III, a differentiator can be derived using the designed observer. Let us consider a smooth signal $y(t)$. It is aimed to estimate the successive time derivatives of $y(t)$ up to the order $(n-1)$, that is to say $\dot{y}(t), \ldots$, $y^{(n-1)}(t)$. Assume that $y^{(n)}(t)=\theta\left(\dot{y}(t), \ldots, y^{(n-1)}(t)\right)$. Set $Y=\left[\begin{array}{llll}y & \dot{y} & \ldots & y^{(n-1)}\end{array}\right]^{T}$. Then

$$
\begin{aligned}
\dot{Y} & =A Y+\Theta(Y) \\
y & =C Y
\end{aligned}
$$

where $(A, C)$ are given in (6) and

$$
\Theta(Y)=\left[\begin{array}{c}
0 \\
\vdots \\
0 \\
\theta\left(\dot{y}(t), \ldots, y^{(n-1)}(t)\right)
\end{array}\right] \in \mathbb{R}^{n}
$$

According to Section III, one can propose the following homogeneous differentiator

$$
\begin{aligned}
& \dot{z}_{1}=z_{2}-k_{1}\left\lfloor z_{1}-y\right\rceil^{\alpha}, \\
& \quad \vdots \\
& \dot{z}_{i}=z_{i+1}-k\left\lfloor z_{1}-y\right\rceil^{i \alpha-(i-1)}, i=2, \ldots, n-1 \\
& \quad \vdots \\
& \dot{z}_{n}=-k_{n}\left\lfloor z_{1}-y\right\rceil^{n \alpha-(n-1)} .
\end{aligned}
$$

Setting $e=Y-z$, one obtains

$$
\begin{aligned}
\frac{d e_{1}}{d t} & =e_{2}-k_{1}\left\lfloor e_{1}\right\rceil^{\alpha} \\
\frac{d e_{2}}{d t} & =e_{3}-k_{2}\left\lfloor e_{1}\right\rceil^{2 \alpha-1} \\
& \vdots \\
\frac{d e_{n}}{d t} & =\theta\left(\dot{y}(t), \ldots, y^{(n-1)}(t)\right)-k_{n}\left\lfloor e_{1}\right\rceil^{n \alpha-(n-1)} .
\end{aligned}
$$

Here due to the term $\theta\left(\dot{y}(t), \ldots, y^{(n-1)}(t)\right)$, it is impossible with this structure to get the convergence of the error to zero without any additive knowledge about the signal and thus the term $\theta\left(\dot{y}(t), \ldots, y^{(n-1)}(t)\right)$. To overcome this problem, one can assume that $x(t)$ is locally polynomial on a small time interval $\left(\theta\left(\dot{y}(t), \ldots, y^{(n-1)}(t)\right)=0\right)$ and, in that case, it is possible to recover the time derivative. Another way is to assume that $\theta$ is bounded such that $\|\theta\| \leq M$ for all $t$. Then, one needs to dominate $M$ by using a discontinuous term as proposed in the next subsection.

\section{B. Link with the higher order sliding mode differentiator}

It has been seen that the state could be recovered in finite time with the observer (11) if $\alpha \in] \frac{n-1}{n}, 1[$. The limit case when $\alpha=1$ corresponds to the well-known Luenberger observer. Let us investigate the other limit case $\alpha \rightarrow \frac{n-1}{n}$ : in that case $\left\lfloor e_{1}\right\rceil^{n \alpha-(n-1)} \rightarrow \operatorname{sign}\left(z_{1}-y\right)$ which will be used to dominate the bound on $\theta\left(\dot{y}(t), \ldots, y^{(n-1)}(t)\right)$.

In [27], the following $(n-1)$-th robust exact differentiator with finite-time convergence was proposed:

$$
\begin{aligned}
& \dot{z}_{1}=z_{2}-k_{1}\left|z_{1}-y\right|^{\frac{n-1}{n}} \operatorname{sign}\left(z_{1}-y\right), \\
& \quad \vdots \\
& \dot{z}_{i}=z_{i+1}-k_{i}\left|z_{1}-y\right|^{\frac{n-i}{n}} \operatorname{sign}\left(z_{1}-y\right), i=2, \ldots, n-1 \\
& \quad \vdots \\
& \dot{z}_{n}=-k_{n} \operatorname{sign}\left(z_{1}-y\right) .
\end{aligned}
$$

Thus, one can recognize (16) with $\alpha=\frac{n-1}{n}$. In that context, using a differential inclusion setting and some results on homogeneity for such a differential inclusion, another reasoning can be used to show that for sufficiently large $k_{n}$, the error dynamics converge to zero in finite time.

\section{APPLICATION TO CHAOTIC SYNCHRONIZATION}

Several chaotic systems, as the three-dimensional GenesioTesi system [8], the Lur'e-like system or the Duffing equation [13], belong to the class of systems (4-5). In this section, the Chua's system is considered to show the effectiveness of the proposed approach. The great simplicity and considerable robustness have made the Chua s circuit a paradigm to generate chaotic signals [28]. The dynamics of Chua's transmitter is given by the following state equation:

$$
\left\{\begin{array}{l}
\dot{x}_{1}=-\frac{1}{C_{1} R}\left(x_{1}-x_{2}-R h\left(x_{1}\right)\right) \\
\dot{x}_{2}=\frac{1}{C_{2} R}\left(x_{1}-x_{2}+R x_{3}\right) \\
\dot{x}_{3}=-\frac{1}{L}\left(x_{2}+R_{0} x_{3}\right)
\end{array}\right.
$$


where

$$
h\left(x_{1}\right)=G_{2} x_{1}+0.5\left(G_{1}-G_{2}\right)\left(\left|x_{1}+H\right|-\left|x_{1}-H\right|\right)
$$

The output is chosen as $y=x_{1}$. Thus, the Chua's circuit is in a similar form as (4-5). In [7] and [13], the authors designed a step-by-step sliding mode observers to perform finite time synchronization of this chaotic system. However, the estimation is based on a step-by-step procedure using successive filtering values of the so-called equivalent output injections obtained from recursive first order sliding mode observers. The approximation of the equivalent information injections by low pass filters at each step may introduce some delays that could lead to inaccurate estimates or to instability for high order systems. The observer given in Section 11 leads to the finite time synchronization of the Chua's circuit using only continuous output injection.

Let us define the linear change of coordinates $z=T x$ where

$$
T=\left[\begin{array}{ccc}
1 & 0 & 0 \\
\frac{1}{C_{2} R}+\frac{R_{0}}{L} & \frac{1}{C_{1} R} & 0 \\
\frac{1}{L C_{2}}\left(1+\frac{R_{0}}{R}\right) & \frac{R_{0}}{L C_{1} R} & \frac{1}{C_{2} C_{1} R}
\end{array}\right]
$$

The system is transformed into the following Brunosvky canonical form:

$$
\dot{z}=\left[\begin{array}{lll}
-a_{1} & 1 & 0 \\
-a_{2} & 0 & 1 \\
-a_{3} & 0 & 0
\end{array}\right] z+\left[\begin{array}{l}
b_{1} \\
b_{2} \\
b_{3}
\end{array}\right] h\left(x_{1}\right),
$$

where

$$
\begin{aligned}
a_{1} & =\frac{1}{C_{1} R}+\frac{1}{C_{2} R}+\frac{R_{0}}{L} \\
a_{2} & =\frac{1}{L}\left(\frac{R_{0}}{C_{1} R}+\frac{R_{0}}{R C_{2}}+\frac{1}{C_{2}}\right) \\
a_{3} & =\frac{1}{C_{1} R L C_{2}} \\
b_{1} & =\frac{1}{C_{1}} \\
b_{2} & =b_{1}\left(\frac{1}{C_{2} R}+\frac{R_{0}}{L}\right) \\
b_{3} & =\frac{b_{1}}{L C_{2}}\left(1+\frac{R_{0}}{R}\right)
\end{aligned}
$$

The observer is given by

$$
\begin{aligned}
\frac{d \widehat{z}_{1}}{d t} & =-a_{1} x_{1}+\widehat{z}_{2}+b_{1} h\left(x_{1}\right)+k_{1}\left\lfloor z_{1}-\widehat{z}_{1}\right\rceil^{\alpha} \\
\frac{d \widehat{z}_{2}}{d t} & =-a_{2} x_{1}+\widehat{z}_{3}+b_{2} h\left(x_{1}\right)+k_{2}\left\lfloor z_{1}-\widehat{z}_{1}\right\rceil^{2 \alpha-1} \\
\frac{d \widehat{z}_{3}}{d t} & =-a_{3} x_{1}+b_{3} h\left(x_{1}\right)+k_{3}\left\lfloor z_{1}-\widehat{z}_{1}\right\rceil^{3 \alpha-2} \\
y & =\widehat{z}_{1}
\end{aligned}
$$

In the simulations, the numerical values of the Chua's circuit are $C_{1}=10.04 \mathrm{nF}, C_{2}=102.2 \mathrm{nF}, R=1747 \Omega$, $R_{0}=20 \Omega, L=18.8 \mathrm{mH}, G_{1}=-0.756 \mathrm{mS}, G_{2}=-0.409$ $\mathrm{mS}, H=1 \mathrm{~V}$. The gains of the observer have been set as follows: $\alpha=0.7, k_{1}=1000, k_{2}=240, k_{3}=24$.
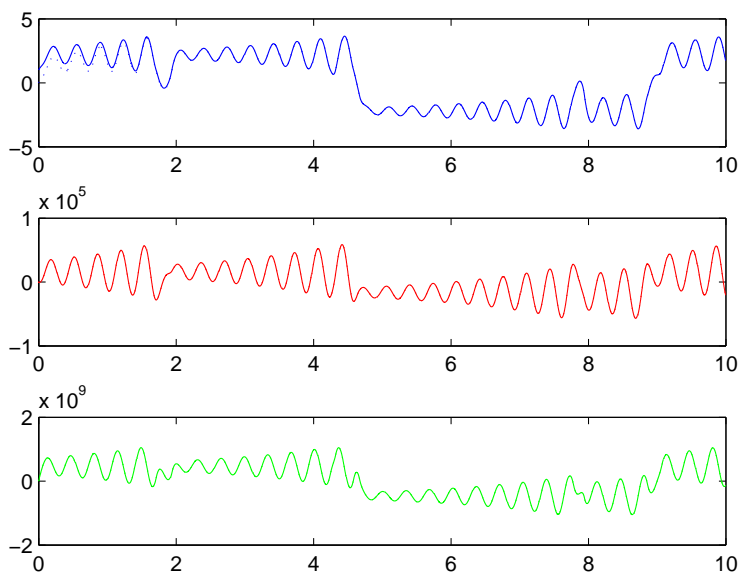

Fig. 1. The $z$ and $\widehat{z}$ time evolution of the chaotic system (21) and its observer (22)
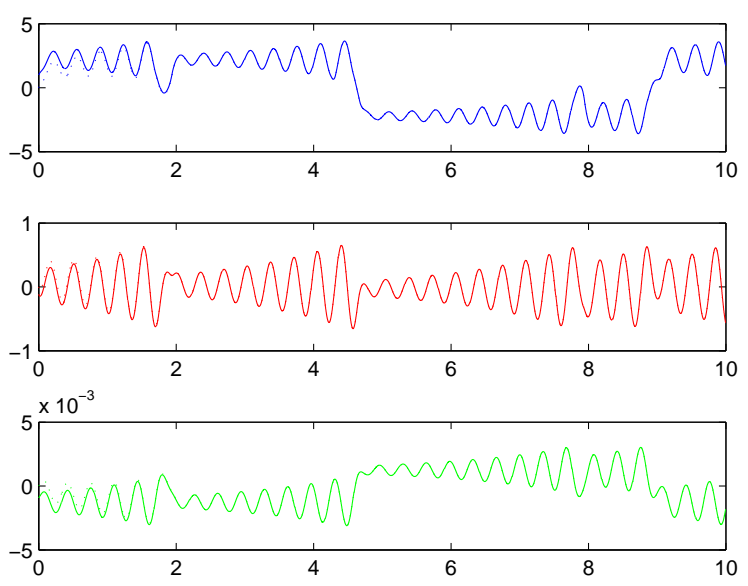

Fig. 2. The $x$ and $\widehat{x}$ time evolution of the chaotic system (19) and its observer (22) with the transformation $T$ (20)

\section{CONCLUSION}

In this paper, a continuous finite time observer based on homogeneity properties has been designed for the observation problem of nonlinear systems that are linearizable up to output injection. It does not involve any discontinuous output injections and step-by-step procedure, as it is the case, for instance, for sliding mode observers. A link with a well-known higher order sliding mode differentiator has been highlighted. Further works aim at extending this result to a larger class of nonlinear systems.

\section{REFERENCES}

[1] Bacciotti A. and Rosier L., Liapunov Functions and Stability in Control Theory, 2nd ed. Springer, Berlin, 2005.

[2] J. Back, K. T. Yu, J. H. Seo, Dynamic observer error linearization, Automatica, Vol. 42, pp. 2195-2200, 2006. 

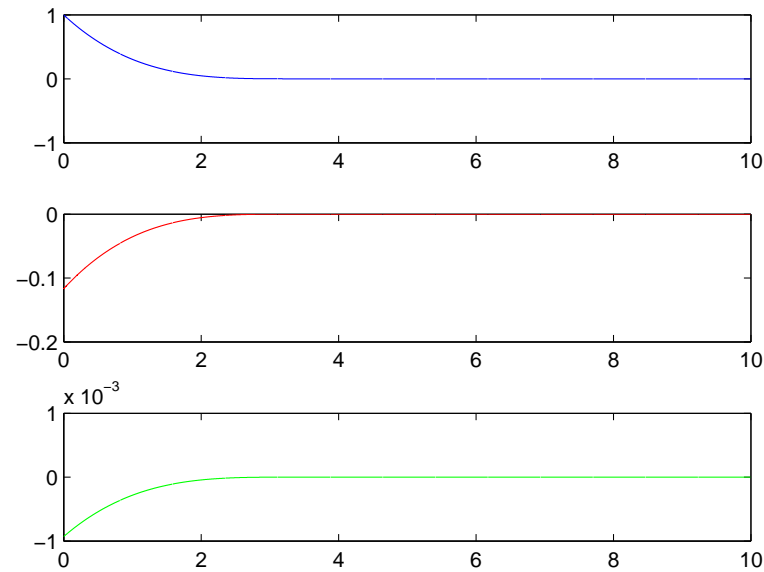

Fig. 3. The error dynamics in the $x$ variables

[3] Bhat S. P. and Bernstein D., Finite-time stability of homogeneous systems. In Proceedings of the American Control Conference, 25132514, Albuquerque, USA, 1997.

[4] Bhat S. P. and Bernstein D., Continuous finite-time stabilization of the translational and rotational double integrator. IEEE Trans. Automat. Control, 43(5):678-682, may 1998.

[5] Bhat S. P. and Bernstein D., Finite time stability of continuous autonomous systems. SIAM J. Control Optim., 38(3):751-766, 2000.

[6] D. Bestle and M. Zeitz, Canonical form observer design for nonlinear time-variable systems, International Journal of control, vol. 38, no. 2, 1983, pp. 419-431.

[7] Boutat-Baddas L., Boutat D., Barbot J.- P., Tauleigne R., Quadratic observability normal form, in IEEE Conf. on Decision and Control, 2001

[8] Chen M. Y., Han Z. Z., Shang Y., General synchronization of GenesioTesi systems, Int. J. Bifurcat. Chaos 14:347-354, 2004.

[9] Chua L, Itoh M, Kocarev L, Eckert K., Chaos synchronization in Chua s circuit, J. Circ. Syst. Computer 1993;3(1):93-108.

[10] S. Diop, J. W. Grizzle and S. Ibrir, On regularized numerical observers, in IEEE Conference on Decision and Control, pp. 2902-2903, 1999.

[11] S. Diop, J. W. Grizzle and F. Chaplais, On numerical differentiation algorithms for nonlinear estimation, in IEEE Conference on Decision and Control, pp. 1133-1138, 2000.

[12] Drakunov S. V. and Utkin V. I., Sliding mode observers. Tutorial, in $34^{t} h$ Conf. on Decision and Control, pp. 3376-3378, 1995.

[13] Feki M., Observer-based exact synchronization of ideal and mismatched chaotic systems. Phys. Lett. A 2003;309:53-60.

[14] A. Glumineau, C. H. Moog, and F. Plestan, New Algebro-Geometric Conditions for the Linearization by Input-Output Injection, IEEE Transactions on Automatic Control, vol. 41, 1996, pp. 598-603.

[15] M. Guay, Observer linearization by output-dependent time-scale transformations, IEEE Transactions on Automatic Control, Vol. 47, pp. 1730-1735, 2002.

[16] Haimo V.T., Finite time controllers, SIAM J. Control Optim., 24(4):760-770, 1986.

[17] R. Hermann and A. J. Krener, Nonlinear controllability and observability, IEEE Transactions on Automatic Control, Vol. 22, pp. 728-740, 1977.

[18] Hermes H., Homogeneous ccordinates and continuous asymptotically stabilizing feedback controls, in Proc. Colorado Springs Conf., Differential equations : Stability \& Control, Marcel Dekker (1990), S. Elaydi Editor.

[19] Hong Y., Finite-time stabilization and stabilizability of a class of controllable systems, Systems Control Lett., 46:231-236, 2002.

[20] Hong Y., Huang J., and Xu Y., On an output feedback finite-time stabilization problem, IEEE Trans. Automat. Control, 46:305-309, 2001

[21] Huijberts H.J.C., Lilge T., Nijmeijer H. Nonlinear discrete-time synchronization via extended observers, Int. J. Bifurcat. Chaos, 11 (7):1997-2006, 2001

[22] Kawski M., Stabilization of nonlinear systems in the plane, Systems Control Lett., 12:169-175, 1989

[23] P. Jouan, Immersion of nonlinear systems into linear systems modulo output injection, SIAM Journal on Control and Optimization, Vol. 41 pp. 1756-1778, 2003.

[24] Kawski M., Geometric homogeneity and stabilization. In Proc. IFAC Nonlinear Control Symposium, 164-169, Lake Tahoe, CA, 1995.

[25] A. J. Krener and A. Isidori, Linearization by output injection and nonlinear observers, Systems and Control Letters, vol. 3, 1983, pp. $47-52$.

[26] A. J. Krener and W. Respondek, Nonlinear observers with linearizable error dynamics, SIAM J. Control Optim., vol. 23, pp. 197-216, 1985.

[27] A. Levant, Higher-order sliding modes, differentiation and outputfeedback control, International Journal of Control, 76, pp. 924-941, 2003.

[28] Madan R.N., Chua's circuit: a paradigm for chaos, In: Chua LO, series editor. World scientific series on nonlinear science. Singapore: World Scientific, 1993

[29] R.L. Mishkov, Non-linear observer design by reduced generalized observer canonical form, International Journal of Control, Vol. 78 , pp. 172-185, 2005.

[30] Moulay E. and Perruquetti W., Finite time stability of differential inclusions, IMA J. Math. Control Inform, 2005.

[31] Moulay E. and Perruquetti W, Finite time stability of non linear systems, In IEEE Conference on Decision and Control, 3641-3646, Hawaii, USA, 2003.

[32] Nijmeijer H. and Mareels I. M. Y., An observer looks at synchronization, IEEE Trans. on Circuits and Systems-1: Fundamental theory and Applications, 44(10):882-891, 1997.

[33] Orlov Y., Finite time stability of homogeneous switched systems, in IEEE Conference on Decision and Control, pages 4271-4276, Hawaii, USA, 2003.

[34] Orlov Y. (2005), Finite-time stability and robust control synthesis of uncertain switched systems, SIAM Journal on Optimization and Control, 43, 1253-1271.

[35] W. Perruquetti, T. Floquet, P. Borne, A note on sliding observer and controller for generalized canonical forms, IEEE Conf. on Decision and Control, Tampa, Florida, USA, 1998

[36] W. Perruquetti and S. Drakunov, Finite time stability and stabilisation, In IEEE Conference on Decision and Control, Sydney, Australia, 2000.

[37] Praly L., Generalized weighted homogeneity and state dependent time scale for linear controllable systems, In IEEE Conference on Decision and Control, pages 4342-4347, San Diego, California USA, 1997.

[38] W. Respondek, A. Pogromsky, H. Nijmeijer, Time scaling for observer design with linearizable error dynamics, Automatica, Vol. 40, No. 2, pp. $277-285,2004$

[39] J. Reger, H. Sira-Ramirez and M. Fliess, On non-asymptotic observation of non-linear systems, Proc. CDC-ECC'05, Sevilla, 2005.

[40] H. Saadaoui, N. Manamanni, M. Djemaï, J.-P. Barbot and T. Floquet, Exact differentiation and sliding mode observers for switched Lagrangian systems, Nonlinear Analysis Theory, Methods \& Applications, Vol. 65, No 5, pp. 1050-1069, 2006.

[41] R. H. C. Takahashi and P. L. D. Peres, Unknown input observers for uncertain systems: a unifying approach, European Journal of Control, 5:261-275, 1999

[42] X. H. Xia and W. B. Gao, Nonlinear observer design by observer error linearization, SIAM J. Control Optim., Vol. 27, 1989, pp. 199-216. 